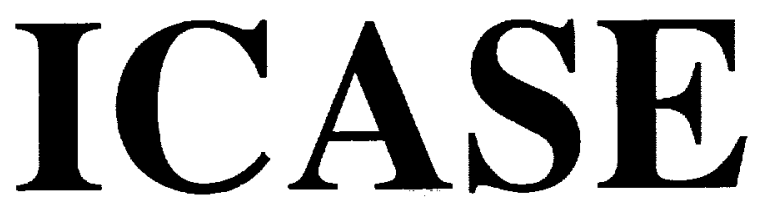

\title{
COUPLING BETWEEN PLATE VIBRATION AND ACOUSTIC RADIATION
}

\section{Abdelkader Frendi}

Lucio Maestrello

Alvin Bayliss

NASA Contract Nos. NAS1-18605 and NAS1-19480

December 1992

Institute for Computer Applications in Science and Engineering NASA Langley Research Center

Hampton, Virginia 23681-0001

Operated by the Universities Space Research Association

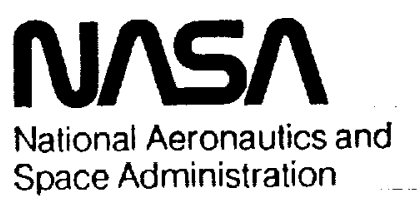

Langley Research Center

Hampton, Virginia 23665-5225 


\title{
COUPLING BETWEEN PLATE VIBRATION AND ACOUSTIC RADIATION
}

\author{
Abdelkader Frendi ${ }^{1}$ \\ Analytical Services and Material Inc. \\ Hampton, VA 23666 \\ Lucio Maestrello \\ NASA Langley Research Center \\ Hampton, VA 23681-0001 \\ Alvin Bayliss ${ }^{2}$ \\ Northwestern University \\ Chicago, IL 60035
}

\begin{abstract}
A detailed numerical investigation of the coupling between the vibration of a flexible plate and the acoustic radiation is performed. The nonlinear Euler equations are used to describe the acoustic fluid while the nonlinear plate equation is used to describe the plate vibration. Linear, nonlinear, and quasi-periodic or chaotic vibrations and the resultant acoustic radiation are analyzed. We find that for the linear plate response, acoustic coupling is negligible. However, for the nonlinear and chaotic responses, acoustic coupling has a significant effect on the vibration level as the loading increases. The radiated pressure from a plate undergoing nonlinear or chaotic vibrations is found to propagate nonlinearly into the far-field. However, the nonlinearity due to wave propagation is much weaker than that due to the plate vibrations. As the acoustic wave propagates into the far-field, the relative difference in level between the fundamental and its harmonics and subharmonics decreases with distance.
\end{abstract}

\footnotetext{
${ }^{1}$ Research was supported by NASA Langley Research Center under NAS1-19317.

${ }^{2}$ Research was supported by the National Aeronautics and Space Administration under NASA Contract Nos. NAS1-18605 and NAS1-19480 while the third author was in residence at the Institute for Computer Applications in Science and Engineering (ICASE), NASA Langley Research Center, Hampton, VA 236810001 .
} 
...

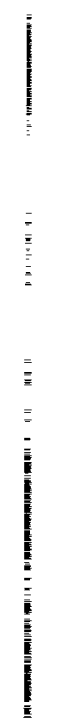

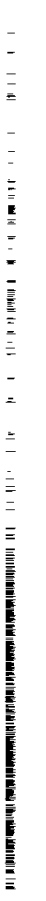




\section{Introduction}

The vibration of a flexible structure excited by an acoustic disturbance has been extensively studied [1-13]. These studies often account for both linear and nonlinear responses, depending upon the amplitude of the excitation mechanism. It has been shown [14] that the coupling between surface motion and the near-field pressure is significant when the surface response is nonlinear, while for a linear response the surrounding pressure field reacts passively with the surface motion. In a recent work Frendi $e t$ al. [15] have studied the problem of a vibrating flexible surface in a supersonic boundary layer. They found that, under reasonable conditions, the vibrating surface could significantly change the structure of the boundary layer over time scales that are long compared to the characteristic time scales of the flow field. The results suggested that the presence of the flexible surface may alter the stability of the boundary layer.

In a wind tunnel experiment, Maestrello and Grosveld [16] found that a flexible plate excited by the combination of boundary layer and sound responded nonlinearly. Recently, Maestrello et al. [17] carried out an experimental and numerical study on the response of a typical aircraft panel to time harmonic, plane acoustic waves at normal incidence. The frequency of the excitation field was chosen to be the fifth natural frequency of the panel. By increasing the amplitude of the excitation source from $110 \mathrm{~dB}$ to $135 \mathrm{~dB}$, the response of the panel changed from linear to chaotic. The route to chaos was found to be through a series of successive period doubling bifurcations. The near-field and far-field pressures were measured and found to behave in a similar way. However, in the far-field a higher level of harmonics relative to the fundamental was observed, which was believed to be the result of nonlinear wave propagation. Using a finite element method, Robinson et al. [18] integrated the nonlinear plate equations subject to a high-intensity random noise in order to predict the plate response. Their numerical results were in good agreement with experiments for the linear case; however, in the nonlinear case the broadening of the spectrum was overestimated. Their model did not account for the coupling to the surrounding acoustic fluid. Mei and Prasad [19] and Prasad [20] used a nonlinear damping 
model in an attempt to explain some experimental phenomena observed for aircraft panels excited by a high intensity sound. They found that the presence of nonlinear damping contributed significantly to the broadening of the response peak at high excitation levels and more realistic RMS deflections.

The problem of nonlinear propagation of acoustic waves emitted by a harmonically vibrating flat plate is of great importance. It was shown by several authors [21-24] that a finite amplitude wave having initially a sinusoidal shape will distort as it propagates. The distortion was described as a compression of the wave front that eventually reaches a point where a shock forms. In the frequency domain, the nonlinear propagation is characterized by the development of high harmonics. This type of behavior has been observed experimentally by Maestrello et al. [17]. A subharmonic route to chaos has also been observed by Lauterborn and Cramer $[25,26]$ in their experiments on acoustical turbulence. Therefore, during propagation both subharmonics and high harmonics are created, leading to the broadening of the spectrum.

Motivated by the results obtained by Maestrello et al. [17] and Robinson et al. [18], a two-dimensional model is developed to study the coupling between the vibration of a flexible flat plate with the surrounding acoustic fluid. The fluid, initially at rest, is excited only by the motion of the plate. The plate is forced at resonance by plane waves at normal incidence. The focus of this paper is to analyze the effect of acoustic coupling (i.e. acoustic damping) on the response of the flexible plate in the various vibration regimes: linear, nonlinear, and chaotic. The problem of nonlinear wave propagation is also addressed.

The remainder of the paper is organized as follows: in the next section, the mathematical model is presented, followed by a description of the numerical methods used. The various results are then discussed, and finally, some concluding remarks are given.

\section{Formulation of the model}

The physical problem addressed in this paper is that of coupling the vibration of a flexible surface with the surrounding acoustic fluid. The governing equations in the acoustic fluid region are the two-dimensional, compressible, nonlinear Euler equations. In 
a cartesian coordinate system, $x$ and $y$, these equations can be written in conservation form as:

$$
Q_{t}=F_{x}+G_{y}
$$

where $Q$ is the vector $(\rho, \rho u, \rho v, E)^{T}, \rho$ is the density, $\rho u$ and $\rho v$ are the $x$ and $y$ momenta respectively, and $E$ is the total energy per unit volume given by

$$
E=\frac{1}{2} \rho\left(u^{2}+v^{2}\right)+\rho c_{v} T
$$

with $c_{v}$ being the specific heat. In eq. (1), the functions $F$ and $G$ are:

$$
F=\left(\begin{array}{c}
\rho u \\
\rho u^{2}+p \\
\rho u v \\
u(E+p)
\end{array}\right)
$$

and

$$
G=\left(\begin{array}{c}
\rho v \\
\rho u v \\
\rho v^{2}+p \\
v(E+p)
\end{array}\right)
$$

In addition to eq. (1), an ideal gas state equation is used:

$$
p=\rho R T
$$

where $p$ is the pressure, $\rho$ the density, $R$ the gas constant, and $T$ the temperature.

The equation describing the motion of the flexible surface is:

$$
D \frac{\partial^{4} w}{\partial x^{4}}-N_{x} \frac{\partial^{2} w}{\partial x^{2}}+\rho_{p} h \frac{\partial^{2} w}{\partial t^{2}}+\Gamma \frac{\partial w}{\partial t}=\Delta p
$$

where $w$ is the plate transverse deflection, $\rho_{p}$ the mass per unit volume of the plate, $h$ the plate thickness, and $\Gamma$ the physical damping. In eq. (5), $D=M h^{3} / 12\left(1-\nu^{2}\right)$ is the stiffness of the plate, with $M$ being the modulus of elasticity and $\nu$ the Poisson ratio of the plate material. The coefficient $N_{x}$ in eq. (5) is given by

$$
N_{x}=\frac{M h}{2 L} \int_{x_{0}}^{x_{0}+L}\left(\frac{\partial w}{\partial x}\right)^{2} d x
$$


which represents the tension created by stretching of the plate due to bending. In eq. (6) $x_{0}$ is the origin of the flexible plate and $L$ its length, see Fig. 1. The forcing term on the right-hand-side of eq. (5) is

$$
\Delta p=p^{-}-p^{+}
$$

where $p^{+}$and $p^{-}$are the pressures on the surface above and below the plate, respectively. It is assumed that there is a pressure wave incident on the upper surface. We have

$$
\begin{gathered}
p^{+}=p_{\infty}+p^{i}+p^{r}+p^{s} \\
p^{-}=p_{\infty}+p^{t}
\end{gathered}
$$

where $p_{\infty}$ is the ambient pressure and $p^{i}, p^{r}, p^{s}$ and $p^{t}$ are the incident, reflected, scattered and transmitted pressure fluctuations, respectively. The scattered pressure is due to the vibration of the flexible surface. Assuming that the fluid on both sides of the plate is the same then the radiated pressure field is anti-symmetric with respect to the plate, therefore

$$
p^{t}=-p^{s}
$$

on the surface of the plate. The input pressure is defined as the sum of the incident and reflected waves and is assumed to be of the form

$$
p^{i}+p^{r}=\epsilon \sin (\omega t)
$$

where $\epsilon$ and $\omega$ are the amplitude and frequency, respectively. The forcing term of eq. (5) can therefore be written as

$$
\Delta p=2 p^{t}-\epsilon \sin (\omega t) .
$$

Equations (1)-(12) are written in a nondimensional form using the following reference quantities for the different variables;

$$
\begin{gathered}
(x, y, w)_{r e f}=l_{r e f}, \quad t_{r e f}=\frac{l_{r e f}}{c_{\infty}}, \quad \text { and } \quad T_{r e f}=\frac{c_{\infty}^{2}}{c_{v}} \\
\rho_{r e f}=\rho_{\infty}, \quad\left(u, v, \frac{\partial w}{\partial t}\right)_{r e f}=c_{\infty}, \quad \text { and } \quad(p, E)_{r e f}=\rho_{\infty} c_{\infty}^{2} .
\end{gathered}
$$


The various freestream fluid properties are those of air at sea-level conditions, which are: temperature $T_{\infty}=288.33^{\circ} \mathrm{K}$, density $\rho_{\infty}=1.23 \mathrm{Kg} / \mathrm{m}^{3}$, pressure $p_{\infty}=1.013 \times 10^{5} \mathrm{~N} / \mathrm{m}^{2}$, and sound speed $c_{\infty}=340 \mathrm{~m} / \mathrm{sec}$. The specific heat at constant volume is $c_{v}=1.004$ $\mathrm{KJ} /\left(\mathrm{Kg}{ }^{\circ} \mathrm{K}\right)$, the ratio of specific heats is $\gamma=c_{p} / c_{v}=1.4$, and the reference length is $l_{\text {ref }}=0.3048 \mathrm{~m}$.

For the results presented here, only the nondimensional quantities are shown on the various graphs. The parameter of particular importance is the nondimensional excitation amplitude $\epsilon^{\star}$, which is given by

$$
\epsilon^{\star}=\frac{\epsilon}{\rho_{\infty} c_{\infty}^{2}}
$$

\section{Method of solution}

The unsteady Euler equations (eq. (1)) are solved using an explicit finite difference scheme. The scheme, which is a generalization of MacCormack's scheme obtained by Gottlieb and Turkel [27], is fourth-order accurate in space and second-order accurate in time. The numerical scheme, applied to a one-dimensional equation of the form

$$
u_{t}=F_{x},
$$

consists of a predictor step given by

$$
u_{i}^{\star \star}=u_{i}^{n}+\frac{\Delta t}{6 \Delta x}\left(-7 F_{i}+8 F_{i+1}-F_{i+2}\right)
$$

followed by a corrector step of the form

$$
u_{i}^{n+1}=\frac{1}{2}\left[u_{i}^{\star \star}+u_{i}^{n}+\frac{\Delta t}{6 \Delta x}\left(7 F_{i}^{\star \star}-8 F_{i-1}^{\star \star}+F_{i-2}^{\star \star}\right)\right] .
$$

In the above equations, the subscript $i$ denotes the spatial grid point and the superscript $n$ the time level. The fourth-order accuracy is obtained by alternating the scheme given above with its symmetric variant. We employ operator splitting to reduce the two-dimensional problem to a sequence of one-dimensional problems. If $L_{x}$ and $L_{y}$ denote the solution 
operators for the one-dimensional $x$ and $y$ problems, then the solution to eq. (1) is obtained by

$$
Q^{n+2}=L_{x} L_{y} L_{y} L_{x} Q^{n}
$$

Further details regarding the method and the advantage of fourth-order schemes can be found in Bayliss et al. $[28,29,30]$.

The boundary conditions employed on the plate for the Euler equations are

$$
v=0 \quad \text { and } \quad T=T_{w}
$$

over the rigid part of the plate, and

$$
v=\frac{\partial w}{\partial t} \quad \text { and } \quad T=T_{w}
$$

over the flexible part of the plate. In eqs. (19) and (20), $T_{w}$ is a specified wall temperature, in this paper it is taken to be the free-stream temperature. The $\mathrm{x}$-component of the velocity $(u)$ is obtained through linear extrapolation from the interior over both the rigid and flexible parts of the surface.

The pressure boundary conditions are as follows: over the rigid part of the surface where the time rate of change of the normal momentum is zero, the pressure is calculated using the normal momentum equation by simply imposing the normal gradient of the sum of pressure and vertical momentum flux to be zero $\left(\frac{\partial}{\partial y}\left(p+\rho v^{2}\right)=0\right)$. Over the flexible part of the surface a linear extrapolation from the interior is used. We first update the pressure to the new time level using $\partial w / \partial t$ at the previous time level, and then, using the new value of $p^{t}$, we solve the plate equation to update $w$. The remaining boundary conditions are derived using the method of characteristics [31]. Radiation boundary conditions [32] have also been used and compared to the characteristic ones. Both boundary conditions gave the same results. We also compared the results obtained using a typical computational domain (see Fig. 1) to that obtained using a much larger domain and found the results to be unchanged. 
The plate equation is integrated using an implicit finite difference method for structural dynamics developed by Hoff and Pahl [33]. The calculation of $N_{x}$ was done using Simpson's rule of integration. The boundary conditions used to solve the plate equation are those for a clamped plate

$$
w=w_{x}=0 \quad \text { at } \quad x=x_{0}, x_{0}+L
$$

The problem of transient oscillations is common when trying to solve the timedependent structural equations. In order to eliminate the transient from the Euler integration, the plate equation is integrated to obtain a steady-state solution without the coupling to the surrounding fluid. The steady-state displacement, velocity, and acceleration profiles are then used as inputs to the Euler calculations to obtain a new steady state.

\section{Results and Discussion}

The numerical experiments simulate the forced vibration of a plate in the presence of an acoustic fluid at sea-level conditions. The properties of the flexible part of the surface are assumed to be independent of position and are: stiffness $D=1.46 \mathrm{~N} \cdot \mathrm{m}$, mass per unit area $\rho_{p} h=2.26 \mathrm{Kg} / \mathrm{m}^{2}$, a physical damping $\Gamma=131.2 \mathrm{~N} \cdot \mathrm{sec} / \mathrm{m}^{3}$, and a Poisson ratio $\nu=0.3$. The plate is $0.254 \mathrm{~m}$ long and $0.0508 \mathrm{~cm}$ thick. Figure 1 shows the computational domain where the top boundary is composed of a flexible surface clamped between two rigid ones. The domain is $6.1 \mathrm{~m}$ long in $\mathrm{x}$ and $3.05 \mathrm{~m}$ wide in $\mathrm{y}$. The number of grid points used in both directions is chosen according to the case being studied. In order to resolve the regions of high gradients near the flexible surface, stretching is used in both directions.

By solving the eigenvalue problem corresponding to the linear unforced vibration of the plate, one can find its natural frequencies. For the plate parameters given above, the first nine natural frequencies are: $45 \mathrm{~Hz}, 122 \mathrm{~Hz}, 206 \mathrm{~Hz}, 396 \mathrm{~Hz}, 591 \mathrm{~Hz}, 826 \mathrm{~Hz}, 1100 \mathrm{~Hz}$, $1413 \mathrm{~Hz}$, and $1765 \mathrm{~Hz}$. Alternate frequencies correspond to symmetric and anti-symmetric modes of the plate. In this paper we consider excitation at $591 \mathrm{~Hz}$. This frequency is chosen because it is close to the frequency used in the experiment (see Maestrello et al. 
[17]). A sequence of solutions are obtained for increasing values of the amplitude of the acoustic excitation $\left(\epsilon^{\star}\right)$. For each value of acoustic excitation, we consider the displacement at the center of the flexible plate, the pressure at the center of the plate $(y=0, x=10.0)$, taken as representative of the near-field pressure, and the far-field pressure at the same $x$ location and at $y=-8.0$. We will present figures illustrating the time history of the selected quantity, the power spectral density (PSD), a phase diagram by plotting the time derivative against the quantity, and a Poincaré map plotting the $(n+1)$ maximum of the time series against the nth maximum. We also present surface plots of the instantaneous pressure field. In addition, we will compare the computed plate response to the response obtained when the coupling to the acoustic fluid is neglected, as is sometimes done in engineering applications.

\section{- Linear Plate Response and Acoustic Radiation}

At first, we consider the case of small amplitude forcing $\left(\epsilon^{\star}=0.0025\right)$ leading to an essentially linear response. The plate response is illustrated by the data presented in Figure 2 for the displacement at the center. The time history and PSD indicate that the response is time periodic and nearly sinusoidal. There is a very weak contribution to the third harmonic, $3 f$; however, this harmonic contribution is not visible in the time history. We note that there is no visible spike at the second harmonic, $2 f$, while there is such a spike for the third harmonic. We believe that this is due to the fact that the frequency $3 f$ is close to the 9 th mode of the plate. This mode is symmetric, and therefore the displacement at the center is nonzero. The phase diagram is almost perfectly circular, as would be expected from the nearly sinusoidal time history, and the Poincaré map reduces to a single point. Comparison with the PSD obtained from the uncoupled calculation indicates that, in this case, the plate response is not significantly altered by accounting for the coupling with the surrounding fluid.

The behavior of the pressure generated by the plate vibrations is illustrated in Figures 3 and 4. In Figure 3, we consider the pressure at a near-field point, while in Figure 4, we consider the pressure at a far-field point. The data in these figures demonstrate that the pressure is time periodic and nearly sinusoidal in both the near field and the far-field. 
In both cases there is no significant harmonic content. The phase diagrams are nearly circular and the Poincaré maps reduce to a point. In Figure 5, we present a surface plot of the instantaneous pressure field. It can be seen from this figure that the pressure exhibits a decaying sinusoidal pattern into the far-field. The radiation is nearly omni-directional when referred to an angle measured from the center of the vibrating plate. We have verified that the RMS of the pressure decays as $1 / r^{1 / 2}$ as expected from linear wave propagation in two dimensions $\left(r^{2}=\left(x-x_{0}-\frac{L}{2}\right)^{2}+y^{2}\right)$.

\section{- Weakly Nonlinear Plate Response and Acoustic Radiation}

We next consider the behavior obtained by increasing the amplitude of the acoustic excitation to $\epsilon^{\star}=0.031$. In Figure 6 , we illustrate the plate response by describing the displacement at the center. The level of the third harmonic is now significantly higher than for the previous case. This increased harmonic content is not directly apparent from the time history; however, it is apparent from the phase diagram, which is no longer circular. The increased harmonic content would be more visible in the plate velocity, $\partial w / \partial t$. The Poincaré map reduces to a single point.

This figure also illustrates a significant effect of the acoustic coupling. The PSD for the uncoupled calculation exhibits enhanced harmonic content together with the generation of noticeable subharmonics. Thus, in the uncoupled calculation, a transition has occurred leading to subharmonic formation $(f / 3$, which is close to the third mode, i.e. the second symmetric mode of the plate). In the fully coupled calculation, there is only a negligible blip at this frequency. The fully coupled response is characteristic of an uncoupled response for a smaller excitation level. Thus, neglecting the coupling of the plate vibration to the surrounding fluid can lead to an overprediction of the plate response at frequencies other than the fundamental.

In Figures 7 and 8 , we describe the pressure generated by the plate vibration in both the near-field (Figure 7) and the far-field (Figure 8). The time history and PSD of the nearfield pressure clearly indicate significant harmonic content, as opposed to the displacement. This is not surprising, as the pressure is obtained from the Euler equations using the normal velocity, $\partial w / \partial t$, as a boundary condition, and differentiating the displacement emphasizes 
the high frequencies. The phase diagram and Poincare map indicate that the near-field pressure is time periodic; however, the nonlinear effects are quite visible in the highly noncircular shape of the phase diagram.

The behavior of the far-field pressure, illustrated in Figure 8, exhibits a more complex dynamical behavior. The time history shows three peaks within each cycle. In addition, the temporal behavior no longer strictly repeats after each cycle. It can be seen that the amplitudes of the $3 f$ and $5 f$ harmonics increase relative to the fundamental as compared to the near-field pressure. The spectral peaks are broader than those in the near-field, which is consistent with the lack of precise periodicity. In addition, the energy in the subharmonic frequency band appears to have increased relative to the fundamental, and some distinct frequency peaks have appeared. It appears that the resulting time history is quasi-periodic with the spectral content generated by the fundamental and a second frequency in the subharmonic frequency band. The frequency resolution is not sufficient to judge whether this additional frequency is rationally related to the fundamental or not. We note that the development of subharmonics as an acoustic disturbance propagates nonlinearly has been observed by Lauterborn and Cramer $[25,26]$. The phase diagram shows three loops, consistent with the three peaks per cycle in the time history. The lines are thicker due to the lack of precise periodicity. Similarly, the Poincaré map shows three closely spaced regions; however, these regions are more spread out than for the linear case due to the lack of precise periodicity.

Another effect of the nonlinear propagation of the acoustic radiation is an enhanced decay with respect to far-field distance. In Figure 9, we plot the RMS of the pressure for $y=-4.0$ and $y=-8.0$ and $x=10.0$ and compare it to the $1 / r^{1 / 2}$ decay characteristic of linear wave propagation in two dimensions. The decay rate is noticeably faster than $1 / r^{1 / 2}$. We also show the corresponding graph for the first case considered, which does exhibit the expected decay rate.

In Figure 10 we present a surface plot of the instantaneous pressure field. This field shows the more complex patterns that develop as the wave propagates nonlinearly. We note that individual ripples appear to break up into multiple ripples, characteristic of the 
generation of high frequencies. There is now a noticeable directivity to the radiation. In

particular, the highest level of radiation occurs along directions that are parallel or nearly parallel to the plate, while the lowest level occurs for directions nearly perpendicular to the plate (measured relative to the center).

\section{- Quasi-Periodic Plate Response and Acoustic Radiation}

When $\epsilon^{\star}$ is increased to 0.032 , multiple peaks develop in the spectrum with levels close to that of the fundamental. This is characteristic of either a complex quasi-periodic or chaotic response. The behavior of the displacement is shown in Figure 11. Computational constraints limit the length of the time samples, and hence of the frequency resolution; however, it appears from the data that the plate response is quasi-periodic rather than chaotic. We note that there are strong frequency spikes at the fundamental and at approximately $200 \mathrm{~Hz}$ and $700 \mathrm{~Hz}$ and that the other noticeable spikes can be obtained as combinations of these frequencies. Our frequency resolution is not sufficient to judge whether these frequencies are rationally related or not; however, it is often the case that quasi-periodic solutions with three incommensurate periods lose stability to chaotic attractors. The phase diagram and Poincaré map are indicative of a quasi-periodic response. We note that for this case there is little qualitative difference between the spectra for the fully coupled and uncoupled cases. However, for the uncoupled response, the spectral peaks are broader and the overall level higher, indicative that a prediction of more complex dynamics would result from a calculation in which the coupling was neglected. We also note that for the uncoupled computation there is a clear shift in the location of some of the frequency spikes, particularly for frequencies greater than the fundamental.

Figures 12 and 13 illustrate the behavior of the near- and far-field pressure respectively. In the near-field, the data appears to indicate quasi-periodic behavior similar to the displacement. We note that while the phase diagram is very complex, there are large regions of phase space that are not visited by the trajectory. Similarly, the Poincaré map indicates localized regions of relatively dense coverings. The time history for the far-field pressure in Figure 13 is qualitatively similar to that of the near-field pressure. The PSD indicates a more pronounced broadband behavior, while the phase diagram shows that a 
larger region of phase space is visited by the trajectory, suggesting more complex dynamics. Similarly, the Poincaré map indicates greater variation in the location of the maxima.

In Figure 14 we plot the instantaneous pressure distribution in the acoustic fluid. The figure shows a marked lack of symmetry in the radiation and the development of multiple peaks in the far-field. We also note the very rapid decay with distance of the pressure peaks close to the plate.

\section{- Nonlinear Plate Response and Acoustic Radiation}

The window where such complex dynamics are found is very small. Upon increasing $\epsilon^{\star}$ further $\left(\epsilon^{\star}=0.0335\right)$, we find the clearly quasi-periodic plate response shown in Figure 15. The time history of the displacement shows that the response is non-periodic; however, each individual cycle is regular and almost sinusoidal. The PSD indicates that the relative level of the $3 f$ harmonic is reduced from the previous nonlinear cases with smaller values of $\epsilon^{\star}$. There is now a clear $f / 3$ subharmonic; however, there is an additional nearby frequency

peak which may account for the non-periodic time history. The most pronounced difference between the spectra obtained from the fully coupled and uncoupled calculations is that the uncoupled calculation exhibits a significantly higher level between spikes and a considerably broader spike at the fundamental. We note that, unlike the previous case, both the fully coupled and uncoupled calculations predict spikes at the same frequencies.

In Figures 16 and 17, we present near- and far-field pressure data for this case. The near-field pressure shown in Figure 16 exhibits clear quasi-periodicity as can be seen from the time history, PSD, phase diagram, and Poincaré maps. We also note the influence of higher harmonics on the time history, as each cycle is no-longer a simple sinusoid. The development of higher harmonics and a broadband spectra in the far-field can be seen from the data in Figure 17. In particular, we note that the trajectory visits a wider area of phase space and the increased level of high frequencies in the PSD.

In Figure 18, we present a surface plot of the instantaneous pressure distribution. We note the development of additional ripples in the pressure field as the waves propagate out to the far-field. In addition, there is a clear directionality in the far-field radiation pattern. 
In this case the near-field exhibits considerably more order than in the previous case (see Figure 14).

\section{Concluding Remarks}

In this paper, we have determined the behavior of a flexible plate using a model that accounts for the full coupling of the plate vibration to the surrounding fluid. For low-level acoustic forcing of the plate, the coupling is weak and need not be considered in making accurate predictions of plate response. For higher amplitude forcing, the effect of the coupling is significant. Generally, the effect of the coupling is to increase the damping on the structure and therefore decrease the response level. Additionally, the coupling can delay subharmonic transitions that occur as the level of the excitation is increased. Thus the response of the plate accounting for acoustic coupling is similar to the response of an uncoupled plate using a reduced level of acoustic excitation. The results show that accurate computation of plate response in the nonlinear regime must account for the coupling with the surrounding fluid.

As the level of acoustic excitation is increased, we find different dynamical responses of the plate. Increasing the excitation level from the linear level leads to a nonlinear response, characterized by the appearance of harmonics in the plate response. Further increase of the excitation level leads to the development of a complex quasi-periodic or chaotic response, characterized by the appearance of both subharmonics and harmonics. Increasing the level still further leads to a reduction in complexity of the plate response, a reduction in the harmonic content of the solution, the development of sharp spectral bands, and distinct subharmonics.

The nonlinear propagation of the acoustic pressure resulting from the nonlinear and chaotic plate vibration has also been studied. Our results show that while the plate response is highly nonlinear, the acoustic wave propagation is weakly nonlinear. This type of propagation is characterized by an increase in harmonic content relative to the fundamental as the distance from the plate increases. In addition, the spectra of the far-field pressure show some broadening of the peaks also characteristic of nonlinear propagation.

The results presented in this paper are in good qualitative agreement with the experimental and numerical results obtained by Maestrello et al. [17]. 


\section{References}

1. W. Y. Tseng and J. Dugundji, 1970. Journal of Applied Mechanics 37(2), 292297. Nonlinear Vibrations of a Beam Under Harmonic Excitation.

2. W. Y. Tseng and J. Dugundji, 1971. Journal of Applied Mechanics 38(2), 467476. Nonlinear Vibrations of a Buckled Beam Under Harmonic Excitation.

3. A. V. Srinivasan, 1966. International Journal of Nonlinear Mechanics 1, 179-191. Nonlinear Vibrations of Beams and Plates.

4. H. R. Busby Jr. and V. I. Weingarten, 1972. International Journal of Nonlinear Mechanics 7, 289-303. Nonlinear Response of a Beam to Periodic Loading.

5. R. Van Dooren and R. Bouc, 1975. International Journal of Nonlinear Mechanics 10, 271-280. Two Mode Sub-Harmonic and Harmonic Vibrations of a Nonlinear Beam Forced by a Two Mode Harmonic Load.

6. N. Yamaki and A. Mori, 1980. Journal of Sound and Vibration 71(3), 333-346. Nonlinear Vibrations of a Clamped Beam with Initial Deflection and Initial Axial Displacement, Part I: Theory.

7. N. Yamaki, K. Otomo, and A. Mori, 1980. Journal of Sound and Vibration 71(3), 333-346. Nonlinear Vibrations of a Clamped Beam with Initial Deflection and Initial Axial Displacement, Part II: Experiment.

8. N. G. R. Iyengar and P. N. Murthy, 1972. Journal of Sound and Vibration 24(1), 93-103. Nonlinear Free Vibration of a Beam with Time Dependent Material Properties.

9. C. Mei and K. Decha-Umphai, 1985. Journal of Sound and Vibration 102(3), 369-380. A Finite Element Method for Nonlinear Forced Vibrations of Beams.

10. E. H. Dowell, 1982. Journal of Sound and Vibration 85(3), 333-344. Flutter of a Buckled Plate as an Example of Chaotic Motion of a Deterministic Autonomous System. 
11. E. H. Dowell and C. Pezeshki, 1986. Journal of Applied Mechanics 53(1), 5-9. On the Understanding of Chaos in Duffings Equation Including a Comparison with Experiment.

12. E. H. Dowell, 1988. Computational Mechanics 3(3), 199-216. Chaotic Oscillations in Mechanical Systems.

13. L. Maestrello, 1969. Journal of Sound and Vibration 10(2), 261-295. Radiation from a Panel Response to a Supersonic Turbulent Boundary Layer.

14. L. Maestrello and T. L. J. Linden, 1971. Journal of Sound and Vibration 16(3), 365-384. Response of an Acoustically Loaded Panel Excited by Supersonically Convected Turbulence.

15. A. Frendi, L. Maestrello, and A. Bayliss, 1992. ICASE Report 92-42. On the Coupling Between a Supersonic Laminar Boundary Layer and a Flexible Surface.

16. L. Maestrello and F. W. Grosveld, 1992. AIAA Journal 30(3), 665-670. Transition Control of Instability Waves Over a Flexible Surface in the Presence of an Acoustic Field.

17. L. Maestrello, A. Frendi, and D. E. Brown, 1992. AIAA Journal, in press. Nonlinear Vibration and Radiation From a Panel with Transition to Chaos Induced by Acoustic Waves.

18. J. Robinson, S. Rizzi, S. Clevenson, and E. Daniels, 1992. Paper AIAA-92-2390, presented at the 33rd Annual Meeting on Structures, Structural Dynamics and Materials Conference organized by AIAA-ASME-ASCE-AHS-ASC. Large Deflection Random Response of Flat and Blade Stiffened Carbon-Carbon Panels.

19. C. Mei and C. B. Prasad, 1987. Journal of Sound and Vibration 117(1), 173186. Effects of Non-linear Damping on Random Response of Beams to Acoustic Loading.

20. C. B. Prasad, 1987. PhD Thesis, Mechanical Engineering Department, Old Dominion University, Norfolk, Virginia. The effects of Nonlinear Damping on the Large Deflection Response of Structures Subjected to Random Excitation. 
21. D. T. Blackstock, 1962. Journal of Acoustical Society of America 34, 9-30. Propagation of Plane Sound Waves of Finite Amplitude in Nondissipative Fluids.

22. A. H. Nayfeh and S. G. Kelly, 1978. Journal of Sound and Vibration 60(3), 371-377. Nonlinear Interactions of Acoustic Fields with Plates Under Harmonic Excitations.

23. J. H. Ginsberg, 1978. Journal of Sound and Vibration 60(3), 449-458. A ReExamination of the Nonlinear Interaction Between an Acoustic Fluid and a Flat Plate Undergoing Harmonic Excitation.

24. M. A. Foda, 1990. Acustica 72(3), 118-130. Analysis of Non-linear Propagation of Waves Induced by a Vibrating Flat Plate.

25. W. Lauterborn and E. Cramer, 1981. Physical Review Letters 47(20), 1445-1448. Subharmonic Route to Chaos Observed in Acoustics.

26. W. Lauterborn and U. Parlitz, 1988. Journal of Acoustical Society of America 86(6), 1975-1993. Methods of Chaos Physics and Their Application to Acoustics.

27. D. Gottlieb and E. Turkel, 1976. Mathematics of Computation 30, 703-723. Dissipative Two-Four Methods for Time Dependent Problems.

28. A. Bayliss, P. Parikh, L. Maestrello, and E. Turkel, 1985. AIAA-85-1694. A Fourth Order Scheme for the Unsteady Compressible Navier-Stokes Equations.

29. A. Bayliss, L. Maestrello, P. Parikh, and E. Turkel, 1986. AIAA Journal 24, 1095-1101. Numerical Simulation of Boundary Layer Excitation by Surface Heating/Cooling.

30. A. Bayliss, L. Maestrello, and E. Turkel, 1981. Journal of Sound and Vibration 74(2), 281-301. On the Interaction of a Sound Pulse with the Shear Layer of an Axisymmetric Jet.

31. S. S. Abarbanel, W. S. Don, D. Gottlieb, D. H. Rudy, and J. C. Townsend, 1990. ICASE Report 90-16. Secondary Frequencies in the Wake of a Circular Cylinder with Vortex Shedding. 
32. A. Bayliss and E. Turkel, 1980. Communications in Pure and Applied Mathematics 33, 707-725. Radiation Conditions for Wavelike Equations.

33. C. Hoff and P. J. Pahl, 1988. Computational Methods in Applied Mechanical Engineering 67, 367-385. Development of an Implicit Method With Numerical Dissipation From a Generalized Single-Step Algorithm for Structural Dynamics. 


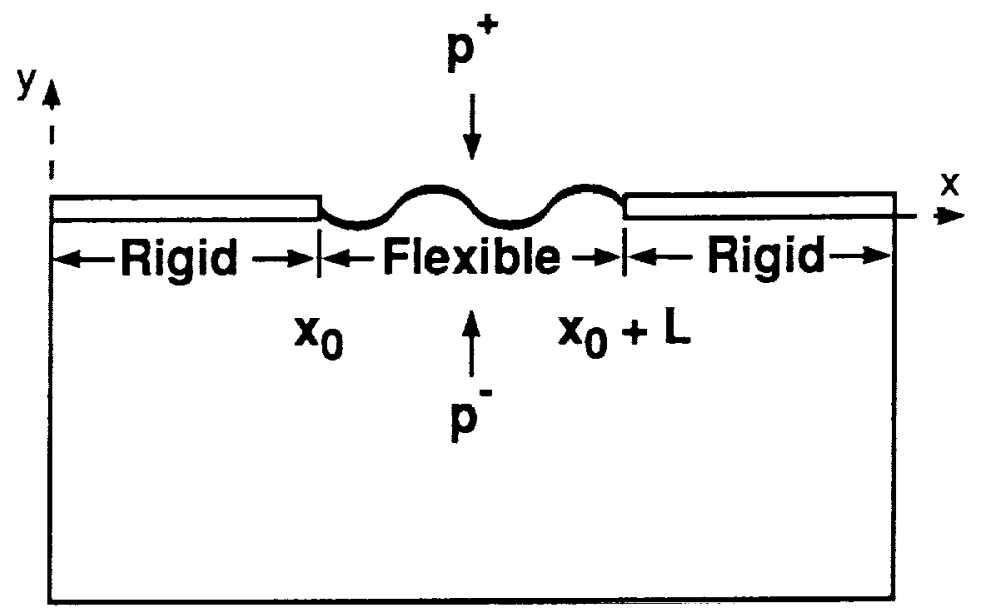

Figure 1: Computational Domain 

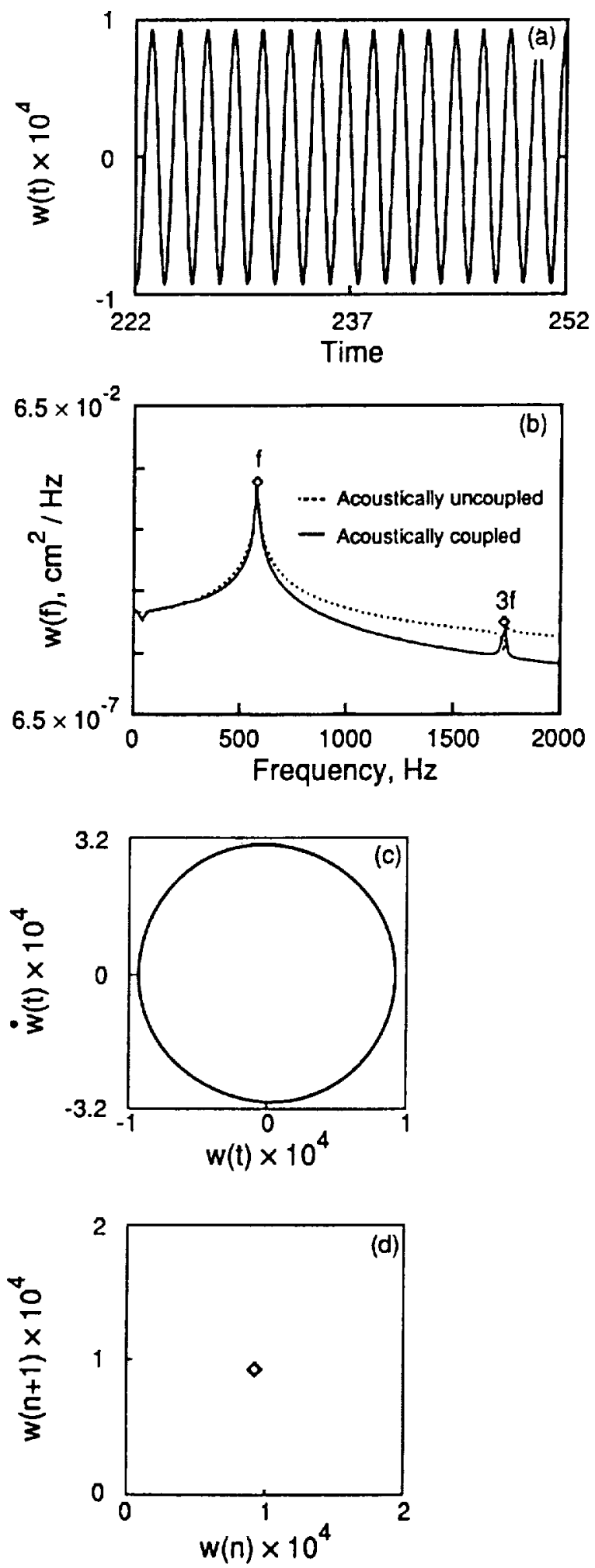

Figure 2: Linear displacement response of the plate center $\left(\epsilon^{\star}=0.0024\right)$. (a) time history, (b) power spectral density, (c) phase diagram, and (d) Poincaré map. 

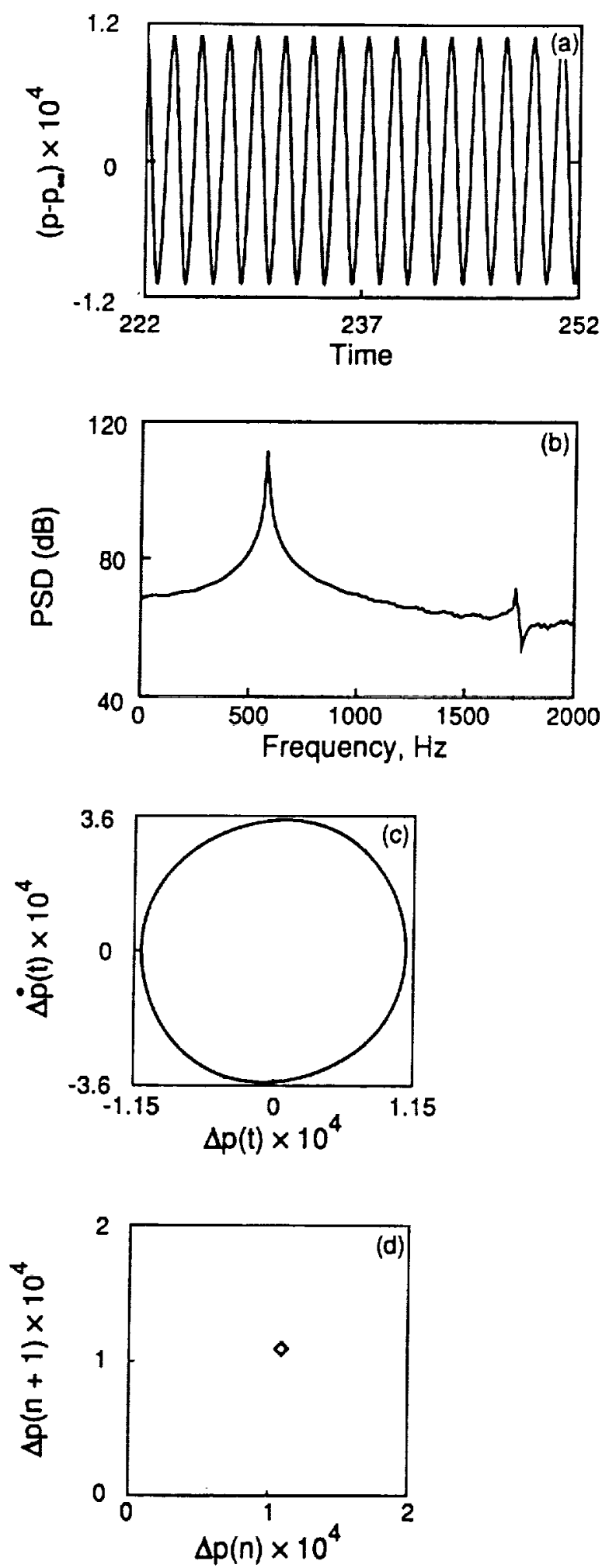

Figure 3: Linear near-field pressure radiated in the direction perpendicular to the plate center $\left(\epsilon^{\star}=0.0024, x=10.0, y=0.0\right)$. (a) time history, (b) power spectral density, (c) phase diagram, and (d) Poincaré map. 

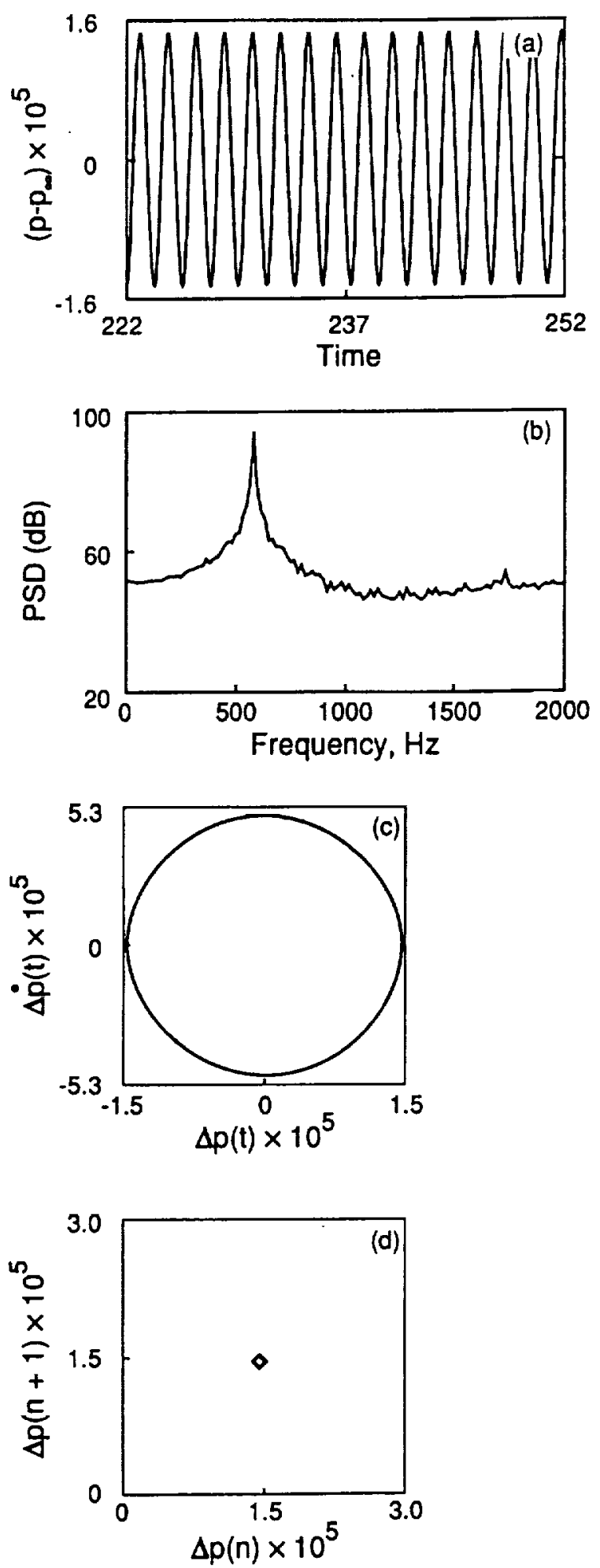

Figure 4: Linear far-field pressure radiated in the direction perpendicular to the plate center $\left(\epsilon^{\star}=0.0024, x=10.0, y=-8.0\right.$ ). (a) time history, (b) power spectral density, (c) phase diagram, and (d) Poincaré map. 


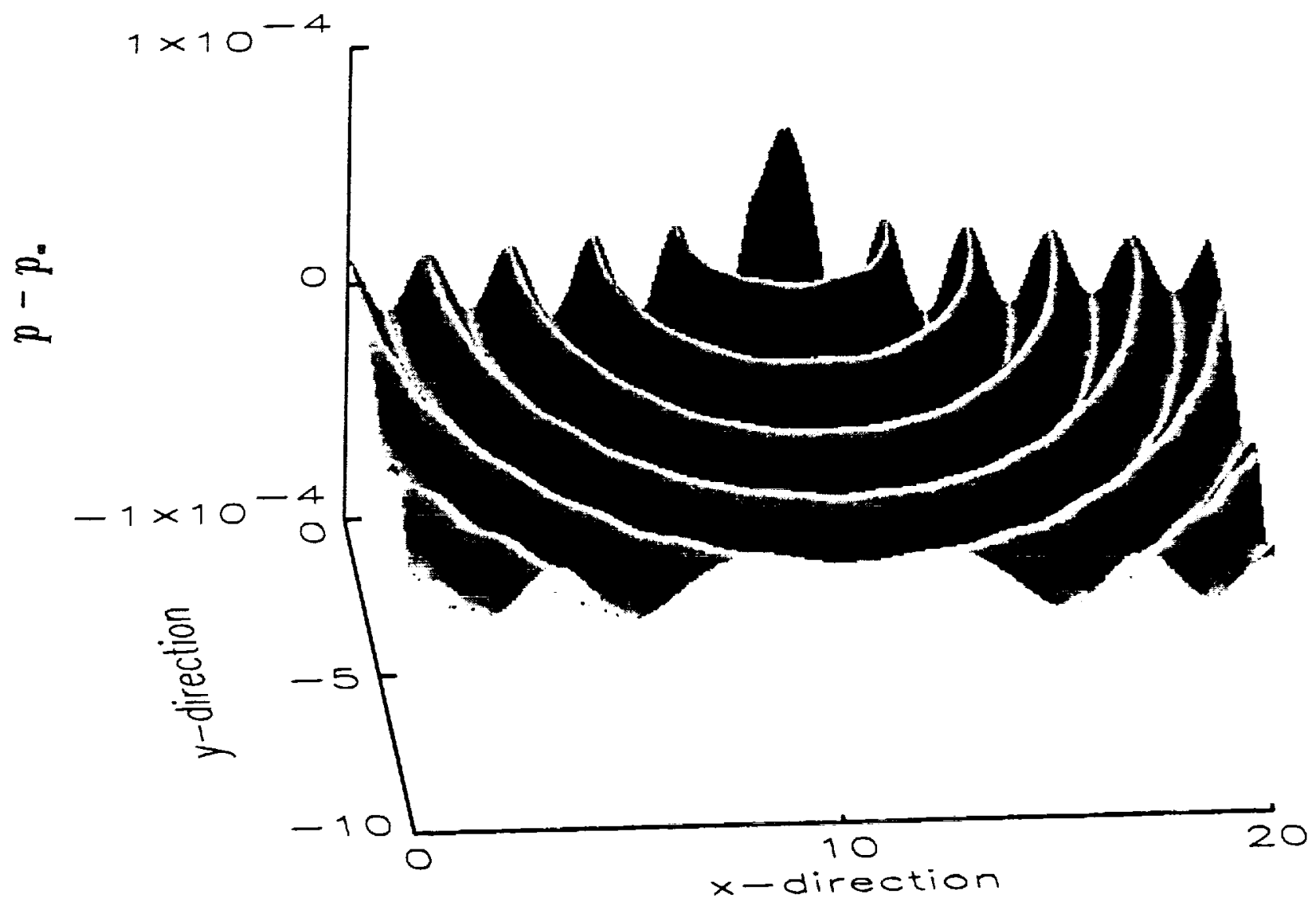

Figure 5: Instantaneous pressure distribution $\left(\epsilon^{\star}=0.0024\right)$. 

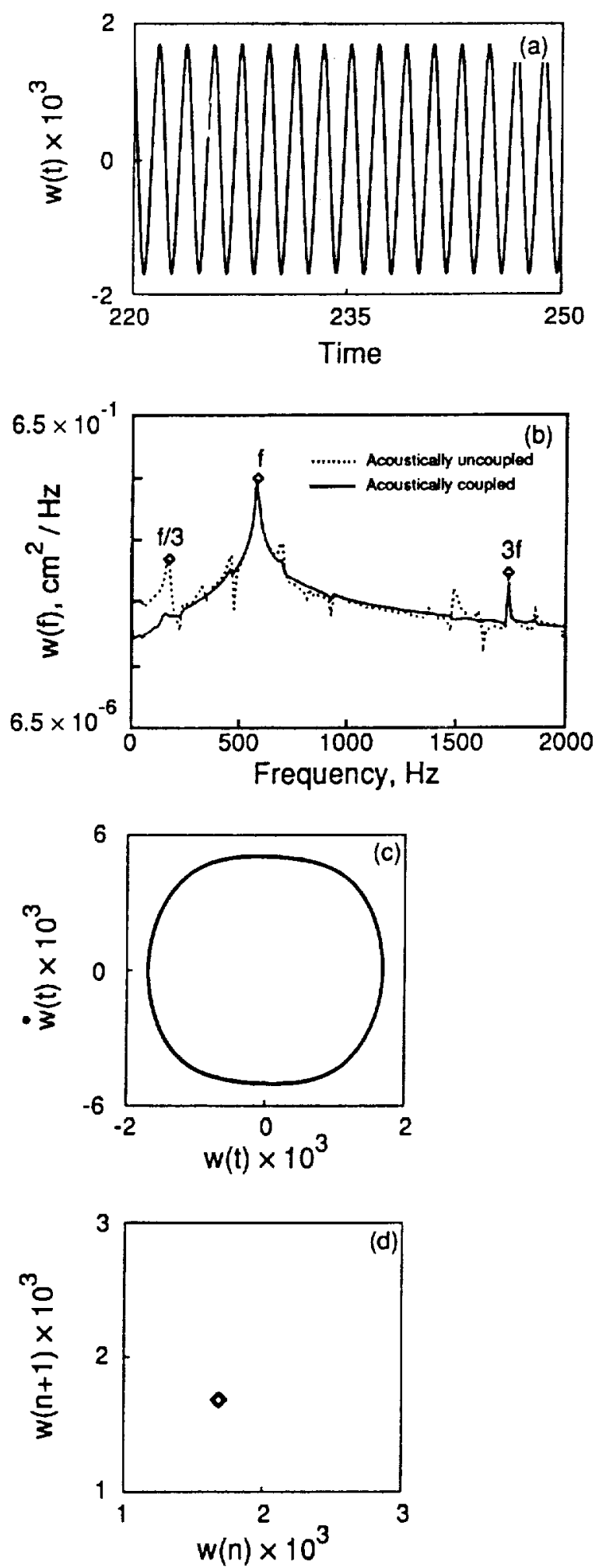

Figure 6: Nonlinear displacement response of the plate center $\left(\epsilon^{\star}=0.031\right)$. (a) time history, (b) power spectral density, (c) phase diagram, and (d) Poincaré map. 

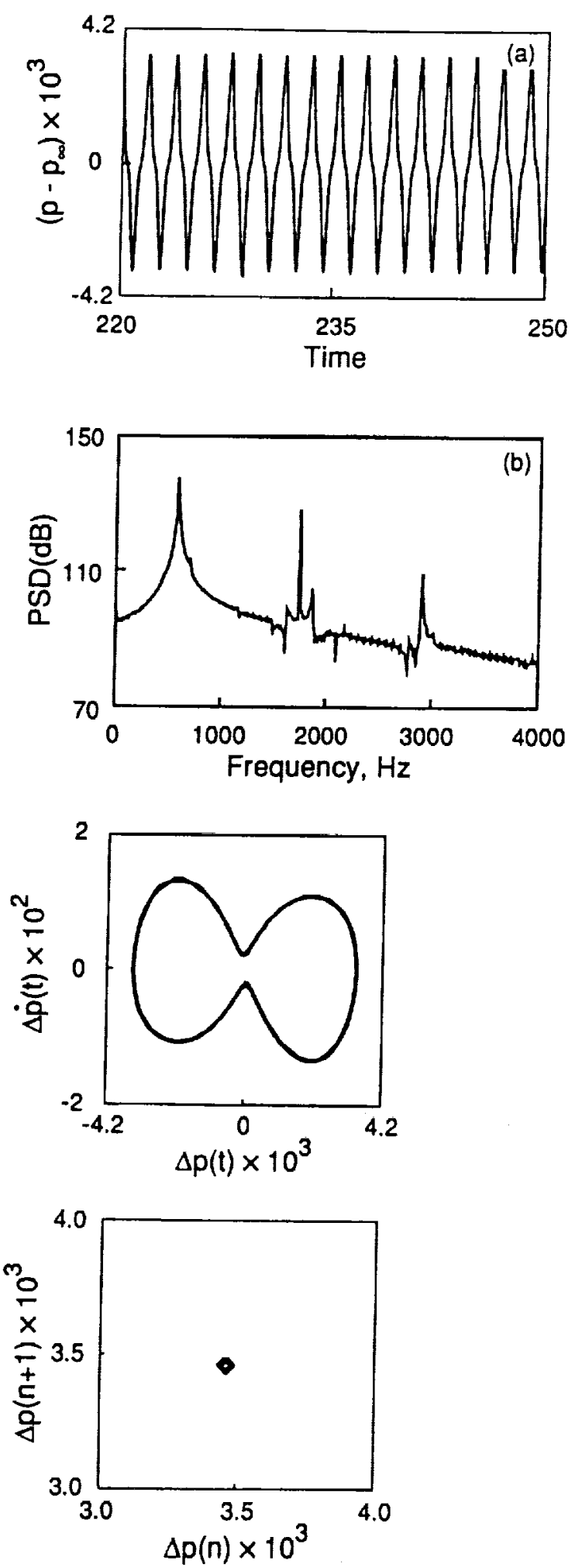

Figure 7: Nonlinear near-field pressure radiated in the direction perpendicular to the plate center $\left(\epsilon^{\star}=0.031, x=10.0, y=0.0\right)$. (a) time history, (b) power spectral density, (c) phase diagram, and (d) Poincaré map. 

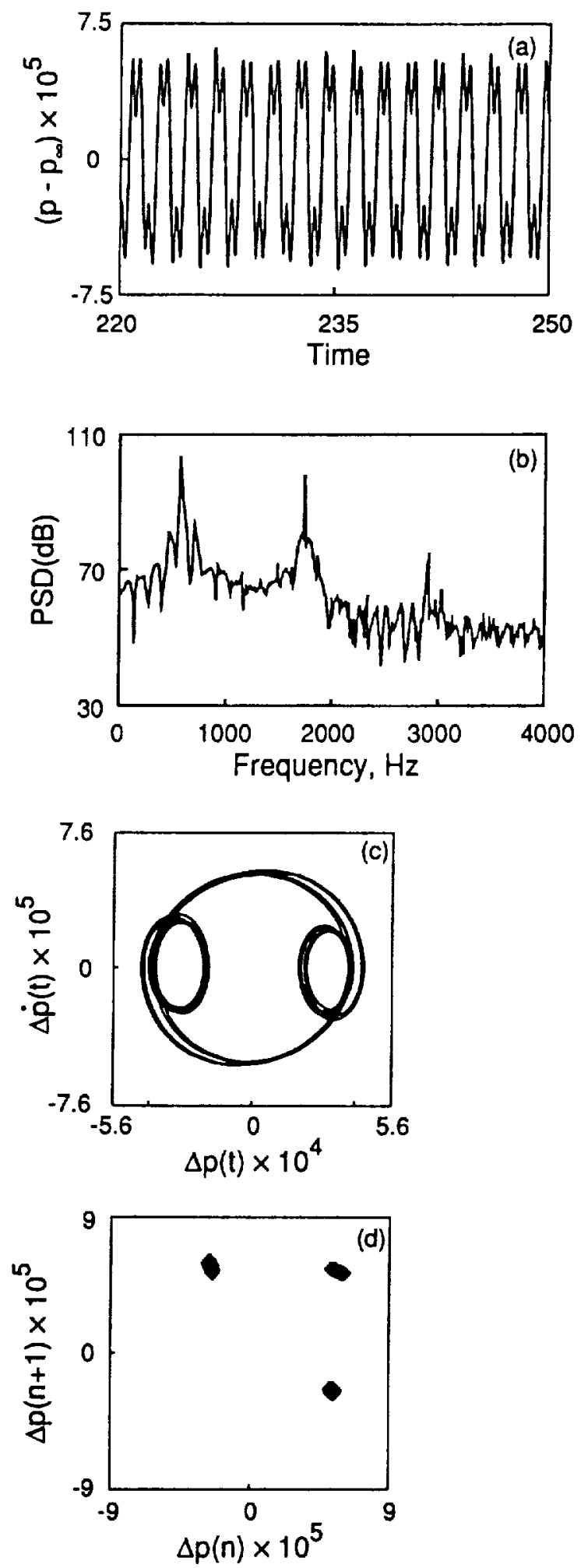

Figure 8: Nonlinear far-field pressure radiated in the direction perpendicular to the plate center $\left(\epsilon^{\star}=0.031, x=10.0, y=-8.0\right)$. (a) time history, (b) power spectral density, (c) phase diagram, and (d) Poincaré map. 

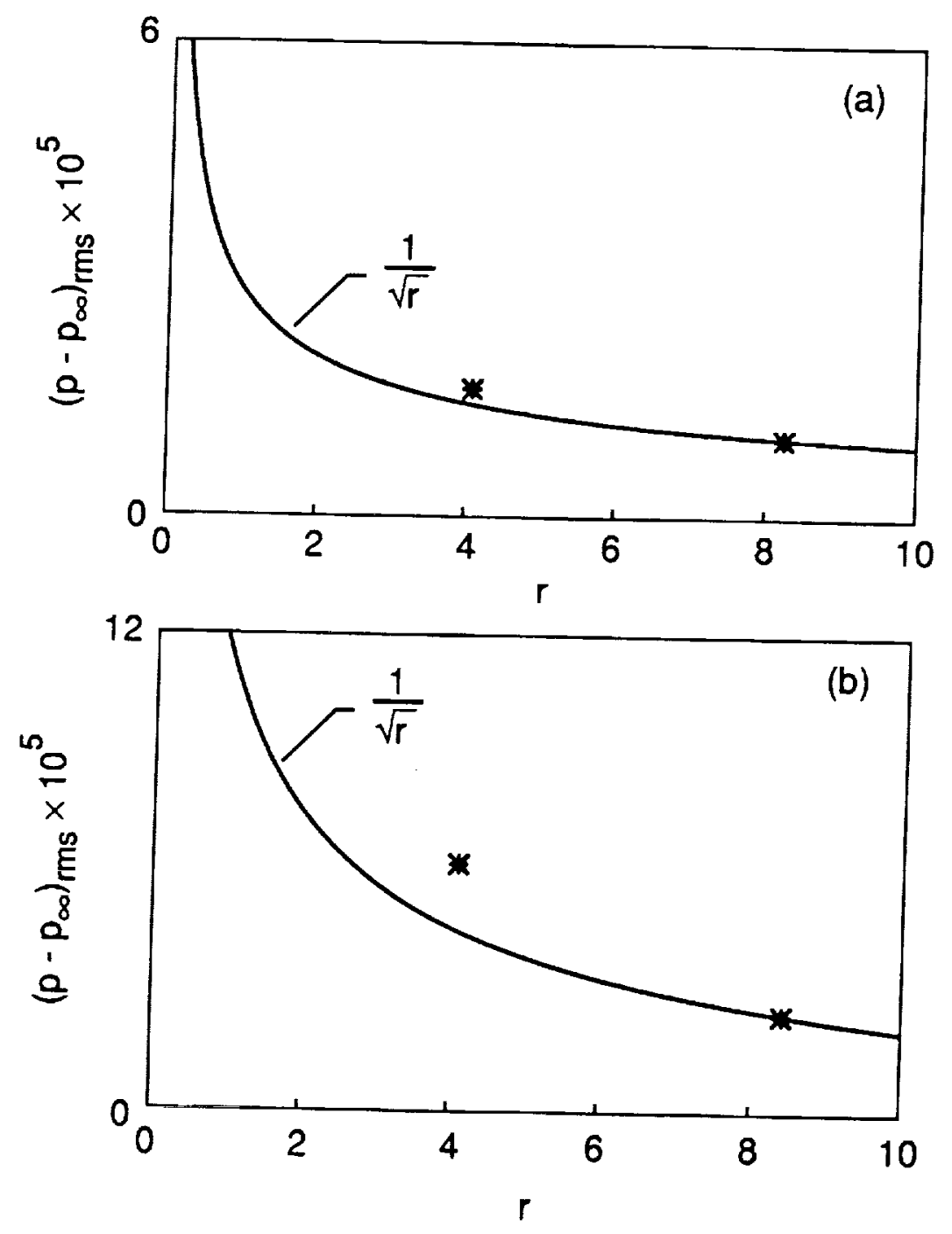

Figure 9: Comparison of the computed RMS pressure with $1 / r^{1 / 2}$ curve. (a) Linear $\left(\epsilon^{\star}=\right.$ $0.0024)$, (b) Nonlinear $\left(\epsilon^{\star}=0.031\right)$. 




Figure 10: Instantaneous pressure distribution $\left(\epsilon^{\star}=0.031\right)$. 

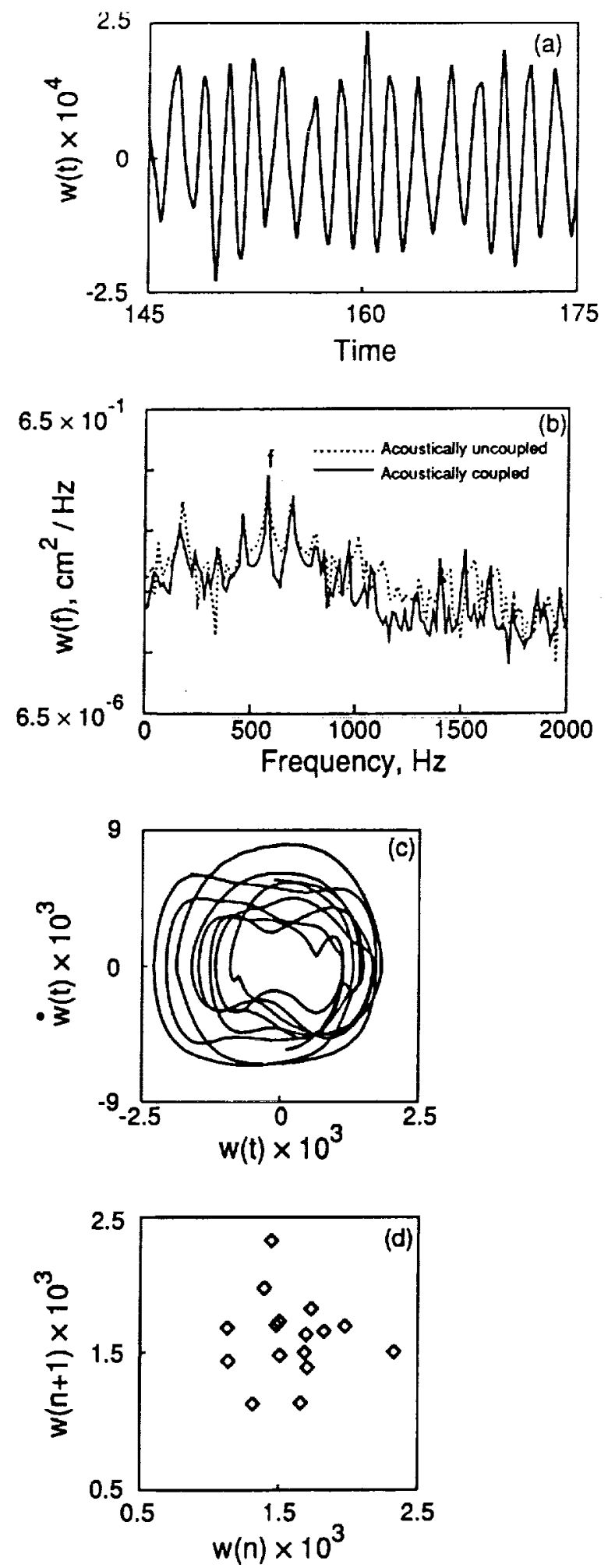

Figure 11: Quasi-periodic displacement response of the plate center $\left(\epsilon^{\star}=0.032\right)$. (a) time history, (b) power spectral density, (c) phase diagram, and (d) Poincaré map. 

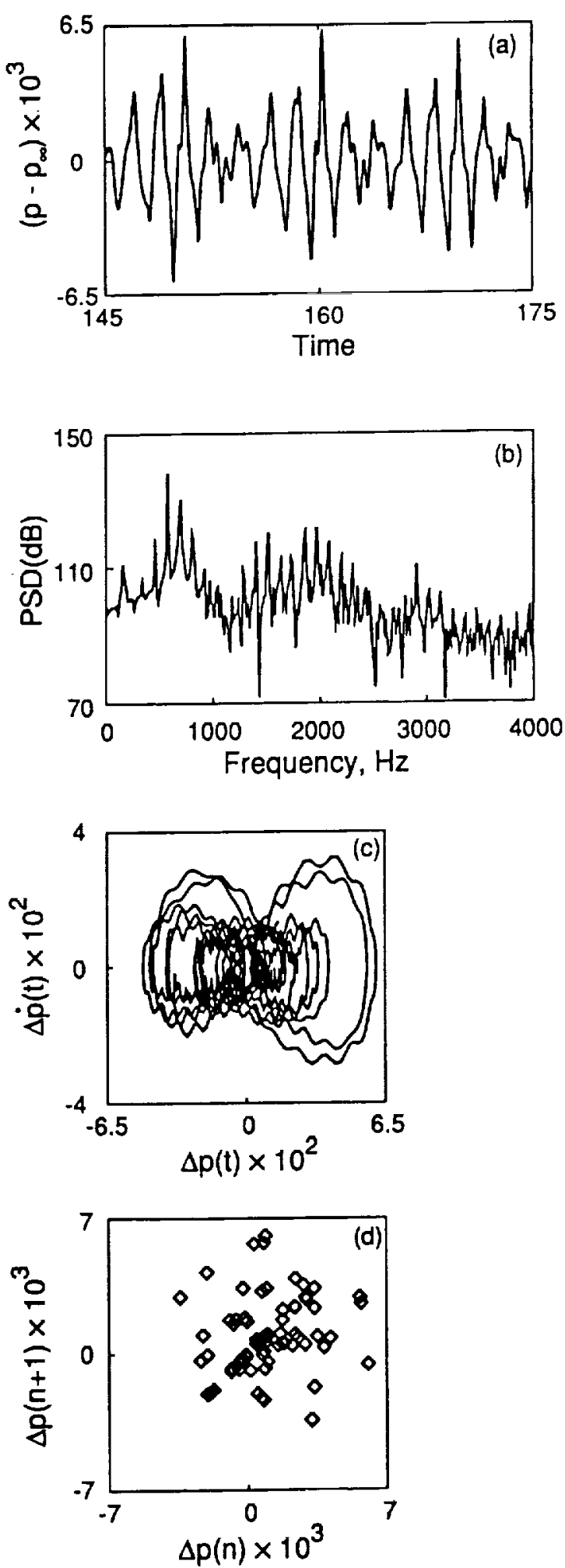

Figure 12: Quasi-periodic near-field pressure radiated in the direction perpendicular to the plate center $\left(\epsilon^{\star}=0.032, x=10.0, y=0.0\right)$. (a) time history, (b) power spectral density, (c) phase diagram, and (d) Poincaré map. 

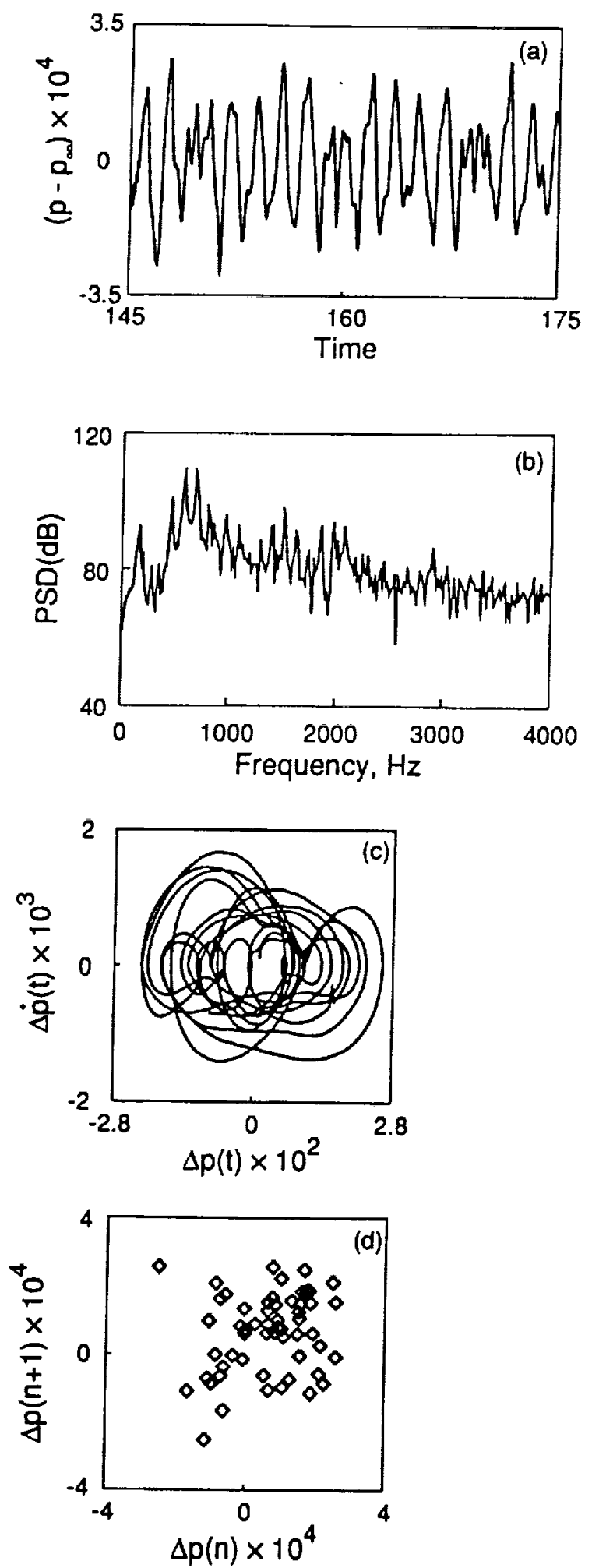

Figure 13: Quasi-periodic far-field pressure radiated in the direction perpendicular to the plate center $\left(\epsilon^{\star}=0.032, x=10.0, y=-8.0\right)$. (a) time history, (b) power spectral density, (c) phase diagram, and (d) Poincaré map. 


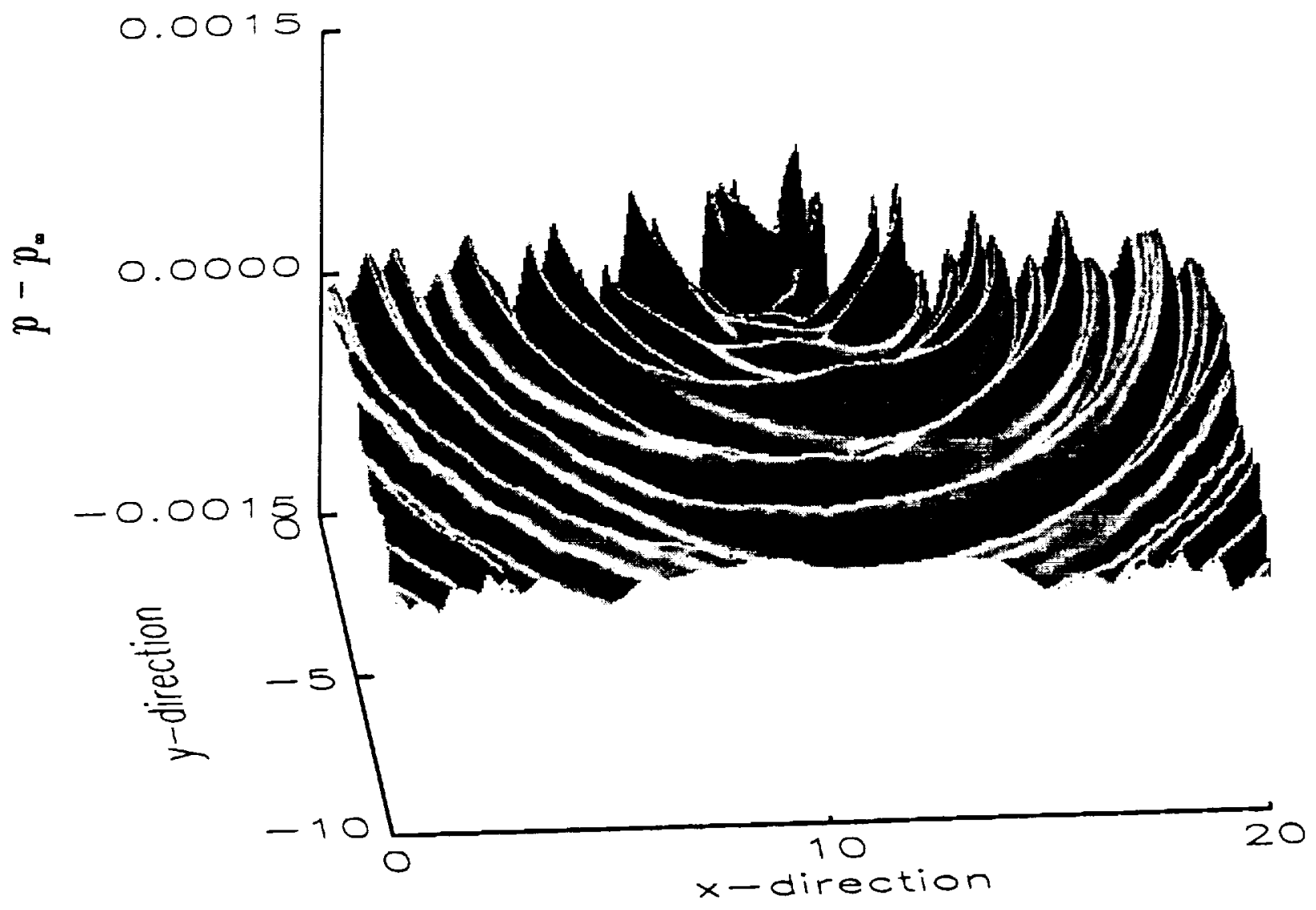

Figure 14: Instantaneous pressure distribution $\left(\epsilon^{\star}=0.032\right)$. 

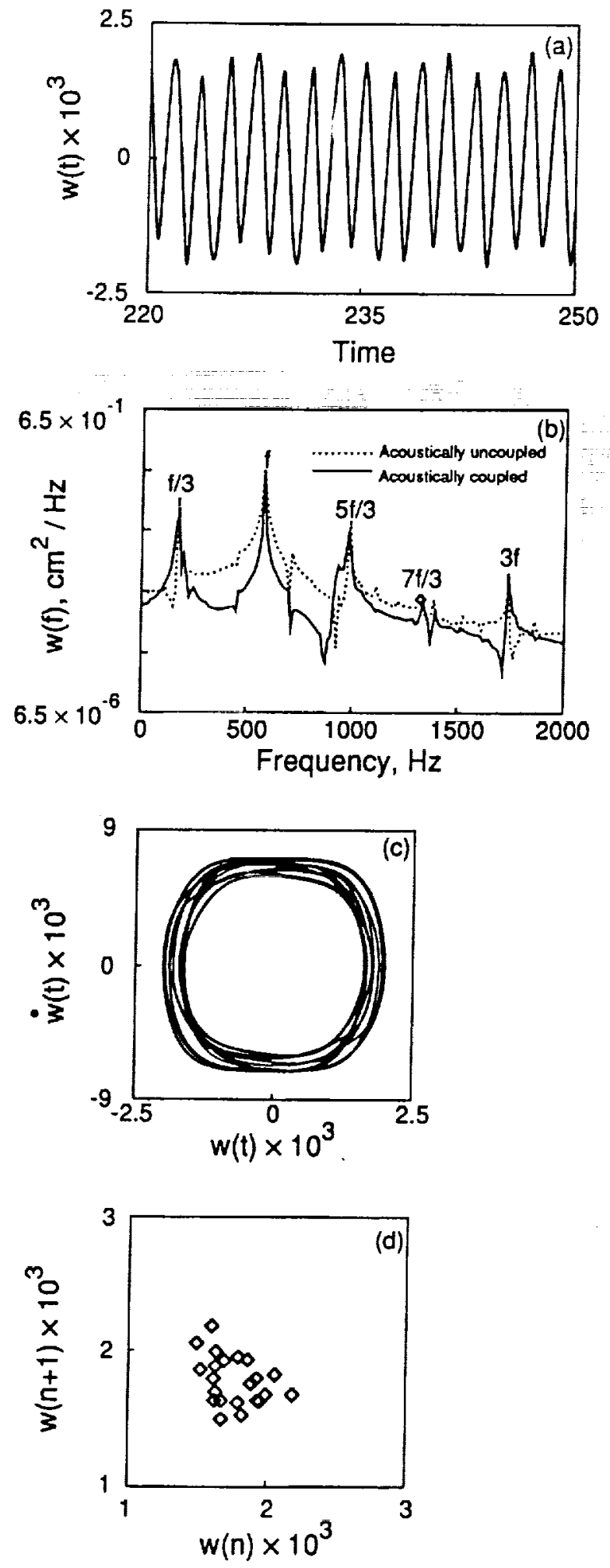

Figure 15: Nonlinear displacement response of the plate center $\left(\epsilon^{\star}=0.0335\right)$. (a) time history, (b) power spectral density, (c) phase diagram, and (d) Poincaré map. 

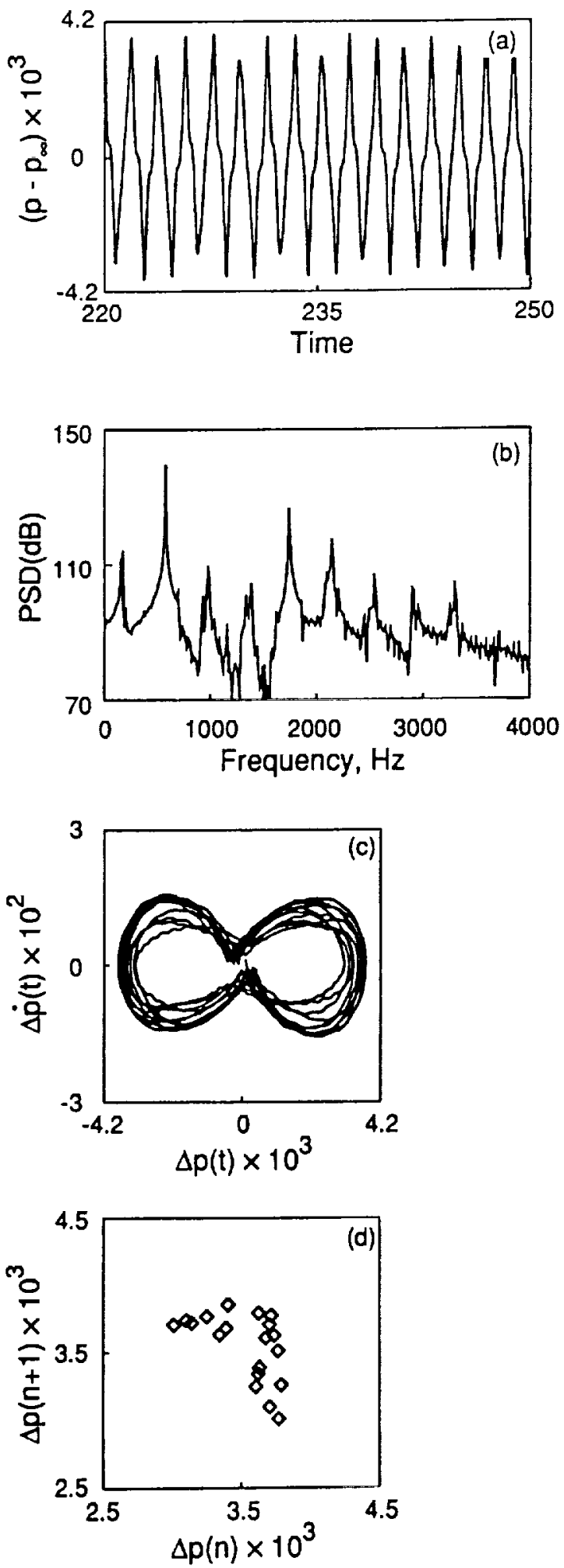

Figure 16: Nonlinear near-field pressure radiated in the direction perpendicular to the plate center $\left(\epsilon^{\star}=0.0335, x=10.0, y=0.0\right)$. (a) time history, (b) power spectral density, (c) phase diagram, and (d) Poincaré map. 

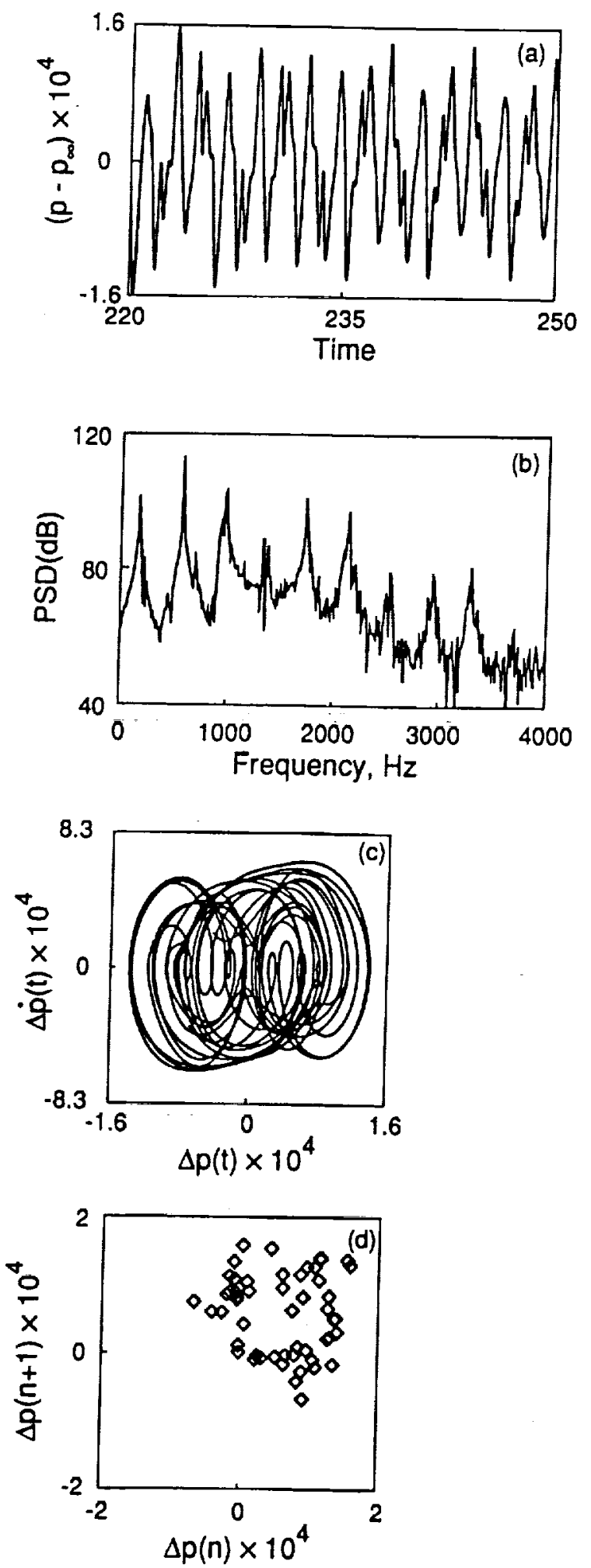

Figure 17: Nonlinear far-field pressure radiated in the direction perpendicular to the plate center $\left(\epsilon^{\star}=0.0335, x=10.0, y=-8.0\right)$. (a) time history, (b) power spectral density, (c) phase diagram, and (d) Poincaré map. 


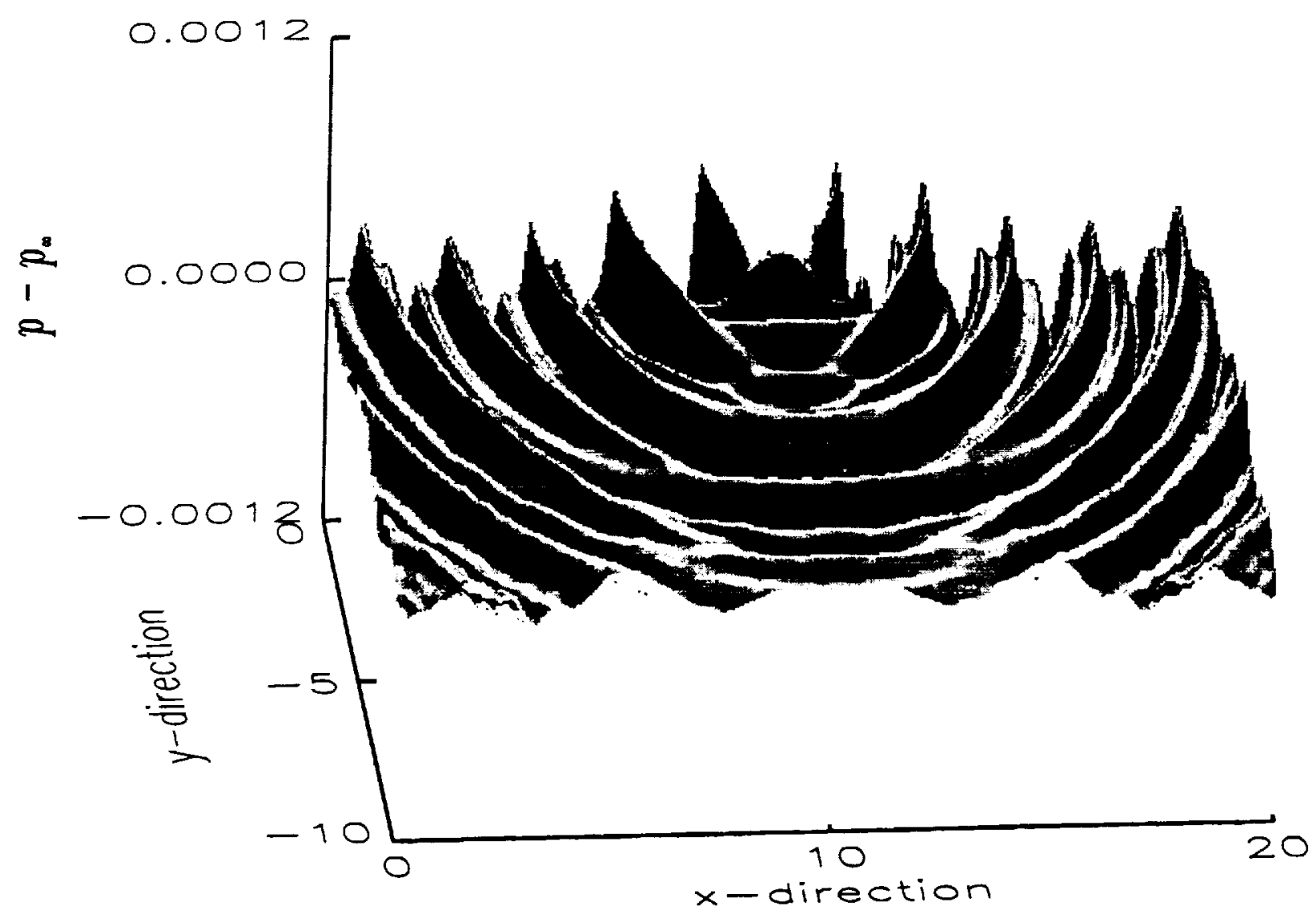

Figure 18: Instantaneous pressure distribution $\left(\epsilon^{\star}=0.0335\right)$. 



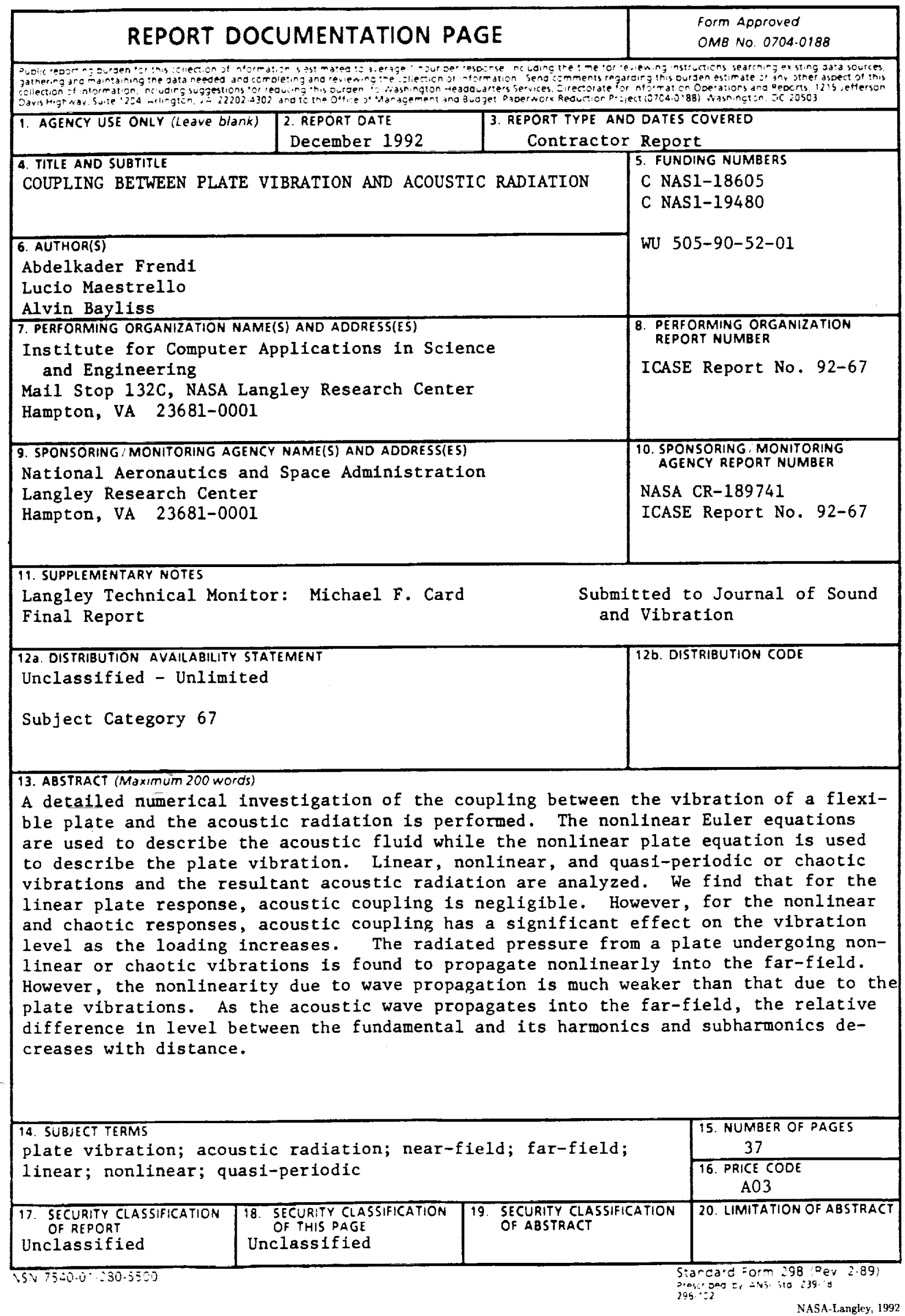

